\title{
A RELEVÂNCIA DO PSICODIAGNÓSTICO NO TRANSTORNO DE PERSONALIDADE BORDERLINE: QUAL O PAPEL DA TCC NO CENÁRIO DA CLÍNICA?
}

\author{
Luciana Santos de Moura ${ }^{\mathrm{I}}$
}

RESUMO: O objetivo desse trabalho é diferenciar o que é a avaliação psicológica do que é o psicodiagnóstico, para que se possa referir sobre a psicopatologia do transtorno de personalidade borderline, a relevância do psicodiagnóstico e os possíveis elementos de enfrentamento na psicoterapia dentro da clínica, com o intuito de minimizar os impactos negativos e prejuízos na vida do paciente, em seu meio familiar e social. Nesse contexto, é apontado a eficácia do tratamento ao utilizar as técnicas e terapias focada no esquema inscrita na abordagem da TCC, evidenciando a importância do manejo qualificado dentro da clínica. Para discorrer sobre o tema foi necessário revisitar várias teorias que apresentam contribuições para o tema, como o Código de Ética dos Profissionais Psicólogos, entre várias outras fontes com o intuito de compreender e colaborar para o construto desse material. Para isso, a metodologia utilizada no presente artigo foi a pesquisa teórica com o intuito de explorar contribuições teóricas de pesquisadores na área.

Palavras-Chave: Avaliação Psicológica. Psicodiagnóstico. Transtorno de personalidade Borderline. Terapia Cognitiva Comportamental. Psicoterapia.

\section{INTRODUÇÃO}

O presente trabalho tem o intuito de à luz das teorias abordar alguns aspectos sobre a psicopatologia do Transtorno de Personalidade Borderline e a partir daí discutir sobre os possíveis meios de enfrentamento no cenário da clínica, enquanto lugar de produção de saberes e prática profissional. Propõe, também, questionar sobre a terapia cognitiva comportamental como melhor intervenção psicoterápica no enfrentamento dos males advindos da TPB, discutindo a sua eficácia diante dos desafios propostos pela referida psicopatologia. A pesquisa teórica utilizada foi de fundamental relevância, uma vez que é uma pesquisa que é "dedicada a recostruir teoria, conceitos, idéias, ideologias,

\footnotetext{
I Psicóloga pela Universidade do Estado de Minas Gerais. Pós-graduada em Avaliação Psicológica e Psicodiagnóstico pelo IBP. E-mail: luhmourapsi@gmail.com.
} 
polêmicas, tendo em vista, em termos imediatos, aprimorar fundamentos teóricos". (Demo, 200o, p. 20)

Inicialmente, o artigo em questão busca convocar à uma reflexão correta, dos profissionais, sobre a diferenciação fundamental entre a avaliação psicológica e o psicodiagnóstico com o intuito de descontruir todo o senso comum entre ambos. Para quê, a partir dessa diferenciação fundamental, seja possível, no âmbito da clínica, desenvolver um trabalho eficaz de psicodiagnóstico frente aos sinais e sintomas de psicopatologias, apresentados como demandas de pacientes, contribuindo assim, ativamente com o processo de minimização do sofrimento e aumento na qualidade de vida. Nesse sentido “o que é fundamental nesta lógica é a compreensão de que as determinantes do enlouquecimento são sócio-históricas e que o aspecto psicológico é que constitui sua variável fundamental, que possibilita sua definição e por isso chama-se psicopatologia.” Havrelhuk e Langaro (2020)

O tema apresentado é de fundamental importância na contemporaneidade e para o profissional psicólogo que atua na clínica, uma vez que o TPB, entre todos os transtornos, é o que mais prevalece causando inúmeros prejuízos biopsicossociais aos portadores. O seu diagnóstico nem sempre é de fácil compreensão ao considerar-se, por exemplo, que tanto o transtorno borderline quanto o transtorno de bipolaridade tem poucas divergências entre si, é necessário que seja apreciado o diferencial teórico e diagnóstico, que no presente caso é o tempo de latência entre os sinais e sintomas entre os dois transtornos. Para tanto, o texto se ancora em pesquisas e argumentações teóricas de alguns autores que se debruçam sobre o assunto.

Por ser uma psicopatologia atual, grave e que têm passado muitas vezes despercebida por familiares e até mesmo por profissionais, o artigo aponta a necessidade de aprofundamento teórico para melhor conhecimento e desconstrução do senso comum, uma vez que, os sinais e sintomas próprios desse transtorno é visto e apontado inúmeras vezes equivocadamente, caindo literalmente no senso comum e consequentemente rotulando o portador de "bipolar, doido, entre outros adjetivos pejorativos" que, ao invés de minimizar o sofrimento tende somente a agravar. 
Além disso, aborda-se que os prejuízos causados são de diversas ordens, tornando, não raras às vezes, o portador incapacitado de ter uma melhor qualidade de vida. Portanto, conhecer a psicopatologia de forma adequada e fazer o seu psicodiagnóstico correto proporcionará não só a prevenção de recaídas, mas a redução de danos e a promoção de saúde em todos os seus aspectos, através de uma intervenção psicoterápica adequada.

Assim, o presente artigo visa compreender inicialmente o transtorno especificado, tendo como ponto de partida sua evolução na história, o psicodiagnóstico correto e os prejuízos biopsicossociais causados. E, nesse ponto, ao considerar a gravidade do transtorno em si, determinar qual é o melhor plano de tratamento e segmento psicoterápico junto ao paciente.

Nesse ponto, pretende-se aqui discutir sobre a intervenção psicoterápica na abordagem da TCC por ser baseada em evidências e por ser uma abordagem diretiva e estruturada, focada nas mudanças de pensamentos e comportamentos disfuncionais, visando consequentemente melhorar a visão de si mesmo (self), do outro e do mundo/futuro (tríade cognitiva), como estratégia de tratamento eficaz no cenário da clínica.

Dessa forma, as reflexões e considerações propostas aqui tem a pretensão de alimentar debates já existentes no meio científico, da eficácia da TCC no cenário da clínica frente a problemática atual e relevante da psicopatologia do TPB. Questiona-se como a TCC se diferencia das demais abordagens no campo de intervenção. Se suas técnicas seriam viáveis na fase aguda da psicopatologia, devido a imprevisibilidade dos comportamentos dos pacientes. Como se configuraria o setting e o manejo terapêutico diante de tal situação crítica. E, o que se pretende dizer sobre a escuta clínica na diferenciação das abordagens de intervenções. São questionamentos como estes que provocaram a construção do presente texto, permeando discussões de aspectos significativos, porém, sem o intuito de encerrar, mas, de se considerar tanto as alterações contextuais e intrapsíquicos quanto as alterações conceituais ao longo do tempo. Para tal construto foi utilizada a pesquisa teórica. 


\section{DESENVOLVIMENTO}

A avaliação psicológica e o psicodiagnóstico são processos interligados e privativos ao profissional psicólogo, entretanto, há uma diferença de extrema relevância entre eles.

A avaliação psicológica é um termo abrangente onde em qualquer contexto ou ambiente uma pessoa pode ser avaliada por um profissional psicólogo e não tem o favorecimento pessoal obrigatório, levando-se a entender que o beneficiário pode ser muitas vezes a outra parte solicitante. Deriva de um processo investigativo com fins de qualificar, quantificar, compreender determinada situação e seus fenômenos psicológicos.

Segundo a Resolução CFP no $007 / 2003$ (2013, p.13), os resultados das avaliações devem considerar e analisar os condicionantes históricos e sociais e seus efeitos no psiquismo, com a finalidade de servirem como instrumentos para atuar não somente sobre o indivíduo, mas na modificação desses condicionantes que operam desde a formulação da demanda até a conclusão do processo de avaliação psicológica.

Já o psicodiagnóstico é uma avaliação psicológica restrita à prática clínica onde o beneficiário é o próprio paciente, constituído por começo, meio e fim, fazendo uso de técnicas, testes psicológicos e outros instrumentos, quando necessários, para fins de investigação de diagnósticos de psicopatologias a partir da demanda prévia apresentada ao profissional. É um processo de avaliação inicial onde se obtém as informações mais relevantes sobre a estrutura psíquica e emocional do paciente e seu dinamismo, identificando os traços de sua personalidade, seu processo criativo em lidar com as diversas situações da vida, e que visa permitir ao profissional psicólogo identificar e compreender amplamente a queixa e o possível transtorno ou desajustamento psíquico provenientes de fatores ambientais e/ou sociais, formulando, a partir daí, possíveis hipóteses diagnósticas mais precisas. Conforme Cunha (2007, p.23) “É um processo que visa a identificar forças e fraquezas no funcionamento psicológico, com um foco na existência ou não de psicopatologia."

A personalidade, que é o padrão de comportamentos e pensamentos, é formada ao longo da vida, desde a infância até o final da vida adulta, sendo influenciada por fatores biopsicossociais atreladas a experiências culturais e sociais, genética, fatores 
neurobiológicos e vivências familiares, ou seja, as contribuições para esse construto são multifatoriais e estão diretamente interligadas entre si.

Entretanto, existem alguns padrões de personalidades que estão atreladas a rigidez e a inflexibilidade, potencializadas à ambientes familiares desestruturados e situações socioeconômicas precárias que levam prejuízos e danos, às pessoas que as detém, trazendo inevitavelmente sofrimentos e desgastes tanto para essas pessoas quanto para àquelas que estão em sua volta. Destarte, quando esses padrões se instalam e se fixam é que podemos dizer que há algum tipo de transtorno de personalidade. Logo,

Para que se possa diferenciar os comportamentos patológicos dos nãopatológicos, dita-se determinadas maneiras de viver como saudáveis e estas são impostas como normas a serem seguidas ou pelo menos almejadas. Isso implica em um exercício de poder pelas ciências sobre os corpos dos indivíduos, que devem passar a seguir as regras de conduta que são respaldadas pelo aval da ciência, ao risco de, se não seguirem, serem apontados como portadores de alguma psicopatologia." Medeiros, Pereira (2021, p.6)

O TPB é considerado um transtorno não normal da personalidade na forma de ver o mundo e a si mesmo, portanto, considerado uma psicopatologia grave. Peculiarmente, possui padrões determinados como instabilidade emocional e afetiva, pensamentos distorcidos e persistentes da realidade, atos impulsivos, sentimentos de desvalia, desamparo e desamor, de menosprezo e solidão, entre outros sentimentos muito intensos não condizente com a realidade e muito negativos, exemplo disso, o sentimento de raiva exacerbada diante de um evento desprazeroso, ainda que em pequena proporção. Essas pessoas vivem na fronteira da instabilidade comportamental e emocional diante das situações vivenciadas, como relações interpessoais e afetivas ou consigo mesmo. A prevalência desse transtorno se dá no sexo feminino, geralmente tendo os primeiros sinais e sintomas na adolescência ou idade adulta. E o seu tratamento, geralmente, se dá através da psicofarmacologia e da psicoterapia associados. Corrobora-se, portanto, que

O Transtorno da Personalidade Borderline - TPB - caracteriza-se por um alto padrão de instabilidade nos aspectos de relações interpessoais, afetos, autoimagem e uma impulsividade intensa. Surge no fim da adolescência/início da vida adulta e geralmente é tratado através de psicofarmacologia e psicoterapia, tendo esta última uma gama de abordagens teóricas diferentes." Silva; Bezerra (202I) 
O termo Borderline foi utilizado pela primeira vez em no século XIX, fazendo referência àqueles pacientes que viviam na fronteira ou no limite da sanidade, entre a neurose e a psicose. Não havia muita precisão, uma vez que, os médicos da época utilizavam esse termo quando se tratava de pacientes com algum sintoma grave da neurose ou da psicose, acreditando que a personalidade não era mutável.

A noção de borderline constitui-se inicialmente como uma entidade vaga e imprecisa, que compreende sintomas que se estendem desde o espectro "neurótico", passando pelos "distúrbios de personalidade", até o espectro "psicótico". Dalgalarrondo e Vilela (1999, p.53). E sendo acrescentado pelo mesmo autor anos depois que "os sintomas começam a surgir no final da infância e adolescência, mais propriamente no começo da vida adulta, podendo durar e permanecer ao longo da vida, sendo relativamente constante durante o ciclo vital com atenuação, sobretudo, da impulsividade e instabilidade afetiva de alguns traços na idade adulta madura e na velhice" (Dalgalarrondo, 2019)

A precisão do termo Borderline veio somente no século $\mathrm{XX}$, mais precisamente em 1980 com o DSM-III, onde a exclusão e a inclusão de critérios específicos para um determinado diagnóstico passaram a ser exigido. O objetivo era

buscar a clareza e afastar possíveis ambiguidades de psicopatologias existentes.

Ainda de acordo com Dalgalarrondo e Vilela (1999, p.53), O conceito atual de borderline foi aquele formulado inicialmente para a classificação norte-americana das doenças mentais de I980, o DMS-III. Neste sistema diagnóstico, a síndrome borderline deixa de ser uma acepção relativamente vaga de estados intermediários neurose-psicose, para ser um distúrbio específico de personalidade, no qual comportamentos impulsivos, auto lesivos, sentimento de vazio interno e defesas egóicas muito primitivas seriam predominantes.

Em relação aos padrões de pensamentos, estudos demonstram que, as interpretações da realidade se tornam distorcidas, coexistindo uma relação direta com a formação da personalidade durante toda a vida da pessoa, dessa forma a rigidez e a inflexibilidade formadas e estabelecidas durante todo esse percurso não permitem novas interpretações ou novas possibilidades de ponto de vista, onde o resultado inevitavelmente será o atrito com aquelas pessoas que estão a sua volta que tem pensamentos, pontos de vistas e/ou opiniões contrárias, trazendo muitos prejuízos e 
efeitos colaterais tanto para o portador do transtorno quanto para as outras pessoas em volta.

E, não menos preocupante, os atos impulsivos como as agressões contra outras pessoas, as automutilações voluntárias e repetitivas, os comportamentos suicidas e de autoextermínio e atos perigosos como o uso de álcool e outras drogas, as compras compulsivas, sexo compulsivo e sem proteção com vários parceiros, o sentimento de vazio intenso, os graves problemas relacionados à identidade e autoimagem são sinais e sintomas recorrentes que não são incomuns em pessoas portadoras desse transtorno de personalidade. "A instabilidade presente nestes indivíduos frequentemente desorganiza a vida familiar, profissional e o próprio senso de identidade do sujeito" (Cavalcanti; Nunes, 2016, p.27)

A instabilidade emocional afetiva sofre uma variedade intensa em pouco espaço de tempo, indo do amor ao ódio na mesma intensidade e proporção, bastando para isso que o parceiro(a) não responda às suas expectativas, tendo como consequência as mais variadas formas de agressões de modo a expressar todo o seu ódio naquele momento. Situações percebidas com mais clareza durante a adolescência, especialmente devido as várias instabilidades emocionais frequentes, presentes nessa fase.

Diante desses fatores pode-se perceber que o transtorno de personalidade de borderline é grave e complexo, que causa prejuízos significativos na vida da pessoa e daquelas que fazem parte do meio, seja familiar, social ou mesmo no ambiente de trabalho. Afirmações que vem corroborar com alguns autores. "O transtorno de personalidade borderline mostra um padrão generalizado de instabilidade $e$ hipersensibilidade em relacionamentos interpessoais" (Muller, 2020) e, "É caracterizado por inúmeros fatores, contudo, nesta revisão sistemática, ressalta-se a dissociação, os sentimentos crônicos de vazio e os conflitos de personalidade." (Usevicius et al, 2020). Tendo, contudo, um agravamento significativo quando se há traumas familiares ou ambientes conturbados e desestruturados durante a infância e a adolescência.

No Manual e Estatístico de Transtornos Mentais - DSM V, o transtorno de personalidade Borderline 301.83 (F6o.3) está representado no grupo B, juntamente com os transtornos da personalidade Antissocial, Histriônica e Narcisista. 
E ainda,

Conforme o DSM-5 (American Psychiatric Association [APA], 2014), o que caracteriza um indivíduo com TPB é um padrão instável no que se refere a relacionamentos interpessoais, autoimagem e impulsividade, que está presente em uma variedade de contextos, tendo início na idade adulta. Alguns estudos indicam que esses pacientes são mais propensos a usar estratégias de enfrentamento de esquiva, mostrando maior intolerância à angústia.

Todavia, mesmo diante do exposto sobre a gravidade dos sinais e sintomas e os reais prejuízos do TPB, na grande maioria das vezes, quando uma pessoa busca a ajuda profissional no âmbito da clínica, não é porque ela por si mesma percebeu que está adoecida ou percebeu a gravidade apresentada pelos sinais e sintomas já explanados acima e seus reais prejuízos biopsicossociais, mas ao contrário, é levada por um familiar ou amigo. Portanto, a adesão e segmento ao tratamento com a psicoterapia nem sempre é aceita imediatamente, e, quando aceita o índice de desistência ou evasão é alto exatamente devido à grande instabilidade do quadro.

Portanto, o bom manejo, os princípios da responsabilidade científica e social, da competência e da integridade, do respeito à dignidade e o bem-estar das pessoas além dos padrões éticos estipulados pelo código, devem estar atrelados no desenvolvimento do trabalho psicológico do profissional. A delimitação cuidadosa desses princípios determinará o resultado satisfatório tanto para o paciente quanto para o profissional.

Segundo os princípios fundamentais do código de ética do profissional psicólogo (2005, p.07): I. O psicólogo baseará o seu trabalho no respeito e na promoção da liberdade, da dignidade, da igualdade e da integridade do ser humano, apoiado nos valores que embasam a Declaração Universal dos Direitos Humanos. II. O psicólogo trabalhará visando promover a saúde e a qualidade de vida das pessoas e das coletividades e contribuirá para a eliminação de quaisquer formas de negligência, discriminação, exploração, violência, crueldade e opressão[...].

Em linhas gerais, o psicodiagnóstico que é um processo científico e que nem sempre visa o fechamento diagnóstico, mas também visa o funcionamento psicológico em suas nuances de força e fraqueza. Assim, alguns autores sugerem que além das técnicas e instrumentos utilizados, como a entrevista aberta, os testes projetivos, o teste de apercepção temática, sejam utilizados também os critérios diagnósticos do DSM-V se 
mantendo como base para realização dessa avaliação, considerando e observando traços que correspondem aos estabelecidos nesse recorte dos critérios.

Assim sendo, de acordo com os critérios diagnósticos do DSM-V, o paciente poderá ser considerado portador do TPB se o mesmo estiver enquadrado em pelo menos cinco ou mais dos supracitados critérios, demonstrando assim um padrão difuso de instabilidade, que é uma característica fundamental e diferencial, em uma grande variedade de nuances, para além de outros conjuntos de sinais e sintomas que se enquadram, descartando o critério isolado, como é apresentado por Aaron Beck.

Ainda segundo Beck (2012, p.187), os pacientes com transtorno de personalidade borderline podem trocar entre quatro modos: O Protetor Desvinculado, A Criança Raivosa, A Criança Abandonada e O Pai Punitivo[...].

Os pacientes portadores do transtorno de personalidade borderline são, não raras às vezes, alvo de preconceitos e rótulos dentro do meio ao qual fazem parte. Viver no limite da sanidade, não é uma opção. O sofrimento e o estrangulamento de uma vida de qualidade são fatores comuns a esses pacientes. Por isso, se faz necessária intervenções terapêuticas pontuais com o objetivo de alterar a rota de todas as visões distorcidas de si mesmo e do outro e de comportamentos disfuncionais frente a eventos adversos. Entretanto, quanto mais rápido for a investigação tão logo será a intervenção adequada com objetivo de cuidar das regulações emocionais e comportamentais.

O setting terapêutico imprescindivelmente precisa ser um espaço aconchegante e seguro, onde o paciente seja capaz de se sentir acolhido e entendido, é onde a relação terapêutica se inicia e se fortalece através de vínculos empáticos, sendo assim, implica-se uma abordagem totalmente voltada às necessidades do paciente para que haja uma sustentação de suporte físico e psíquico do mesmo. É nesse local que é apresentado formas de enfrentamentos, em conjunto, aos males provenientes do TPB.

Dessa forma, pode-se citar que a abordagem de terapia que tem melhores resultados frente ao supracitado transtorno é a terapia cognitiva comportamental, a qual é focada no aqui e agora, tem curta duração, é diretiva e estruturada, requer a participação ativa tanto do paciente quanto do profissional com objetivo nas mudanças de 
comportamentos e pensamentos distorcidos e/ou inadequados, trazendo soluções para problemas pontuais e/ou minimizando os impactos negativos.

A abordagem cognitivo-comportamental vem propondo intervenções eficientes nesse contexto, fazendo com que seja importante a pesquisa $e$ conhecimento aprofundado sobre os métodos de tratamento, promovendo aperfeiçoamento e propiciando novos estudos sobre um transtorno de personalidade tão complexo como o Borderline" SILVA, GARUTTI. COSTA (2017, p.I). E ainda, "A TCC é uma intervenção estruturada, que tem como objetivos: I) identificar pensamentos automáticos negativos e distorções cognitivas, 2) modificar crenças disfuncionais e desenvolver crenças mais adaptativas sobre o próprio e os outros, e 3 ) implementar estratégias comportamentais que promovam melhores níveis de funcionamento." MARQUES, BARROCAS e RIJO (2017, p.309)

Aqui é necessário ressaltar que o bom manejo profissional dentro da clínica está relacionado ao controle das instabilidades emocionais e comportamentais intensas, avaliação de risco próprio do paciente e para terceiros, ao desenvolvimento de habilidades funcionais no enfrentamento de eventos estressores visando a minimização dos danos e prejuízos e a ativa compreensão do sujeito em relação ao seu funcionamento biopsíquico.

Portanto, um grande desafio ao profissional ante uma problemática atual e relevante. É necessário ressaltar que o papel do psiquiatra no enfrentamento a essa psicopatologia tem grande relevância, uma vez que os psicofármacos são fundamentais devido ao alto índice de ideação suicida e outros transtornos afetivos comórbidos nesse grupo de portadores, principalmente durante a adolescência. Sendo assim, um não exclui o outro, mas um complementa o outro, no tocante ao tratamento e manutenção de segmento.

Quando se há adesão, ainda que haja muita resistência no início, ao segmento de tratamento psicoterápico, vários autores concordam que a melhor intervenção terapêutica é a terapia cognitiva comportamental (TCC) que tem como princípio de que a forma como pensamos influencia nossas emoções e os nossos comportamentos, sendo a melhor estratégia para enfrentamento no presente caso, uma vez que é focada no problema atual. E que segundo Adami, Portella e Dias (2020) "Esta terapia baseia-se em princípios, e não em protocolos específicos estabelecidos. É composta pela terapia individual e grupo de treinamento de habilidades."

No presente artigo, o recorte do segmento de tratamento e manutenção do estado geral no transtorno referido, como já explanado, é a terapia cognitiva comportamental "uma abordagem ativa, diretiva, por tempo limitado, estruturada(...) baseada no fundamento lógico teórico subjacente de que o afeto e o comportamento do indivíduo são amplamente determinados pela maneira como ele estrutura o mundo" (Beck et al., 1979). Levando-se em consideração que os distúrbios psicológicos possuem padrões de pensamentos disfuncionais e distorcidos gerando alterações de humor e comportamentos. E ainda,

O principal objeto da terapia cognitiva são os aspectos cognitivos no processamento de informações e atribuições aos seus significados nos processos 
cognitivos entre as emoções e repertórios comportamentais. E, não menos relevante, a aliança terapêutica junto ao embasamento teórico são fundamentais para o bom andamento e resultados satisfatórios para o paciente.

O Dr. Beck começou a ajudar os pacientes a identificar e avaliar esses pensamentos automáticos. Ele descobriu que, ao fazer isso, os pacientes eram capazes de pensar de forma mais realista. Como resultado, eles se sentiram melhor emocionalmente e foram capazes de se comportar de forma mais funcional. Quando os pacientes mudaram suas crenças subjacentes sobre si mesmos, seu mundo e outras pessoas, a terapia resultou em uma mudança duradoura. Dr. Beck chamou essa abordagem de "terapia cognitiva". Também se tornou conhecido como "terapia cognitivo-comportamental" ou "TCC". INSTITUTO BECK (2020)

A TCC é focalizada em problemas com objetivos e metas de extinguir ou minimizar os resultados de comportamentos e pensamentos negativos e disfuncionais diante de eventos estressores, modificando comportamentos/pensamentos desadaptativos e consequentemente promovendo uma melhor qualidade de vida aos portadores de $\mathrm{TPB}$, os quais vivem em uma montanha russa de instabilidades rápidas e agudas, o que gera uma situação social e clínica muito desafiadora.

A dificuldade de regulação emocional presente em portadores de TPB, advém de crenças e valores estabelecidos ao longo da vida, através de uma visão a respeito de si mesmo, dos outros e do mundo, de uma forma disfuncional e desadaptativa. A TCC tem por objetivo o mapeamento e entendimento desses desajustes para regular essas emoções através das mudanças de pensamentos, emoções e comportamentos, da forma desadaptativa para a forma adaptativa.

A valorização das queixas apresentadas ao profissional psicólogo visa não somente as mudanças de aspectos cognitivos em si, mas também as comportamentais e afetivas. É nesse momento, que o profissional busca compreender os sentimentos e pensamentos do paciente, distorcidos e desadaptativos, e de como eles se relacionam para produzir certo comportamento. As crises agudas e imprevisíveis são situações comuns dentro do cenário da clínica e, é nesse momento que o profissional psicólogo é convocado a intervir de forma assertiva e empática, acessando pensamentos automáticos e crenças com o intuito de, através de evidências, trazer o paciente a um contexto mais realista e palpável. A empatia por parte do profissional é fundamental, pois a compreensão desse fenômeno se dá através da auto-observação, do exame de evidências e da determinação das atividades disfuncionais associados aos focos intrapsíquico, interpessoal e externo.

A escuta dentro da abordagem da TCC se diferencia das demais abordagens por ser uma escuta pontual no momento da queixa presente, uma escuta assertiva com o intuito de intervenções psicoterápicas baseadas em evidências. Para além da escuta, a participação é ativa e convoca o paciente a fazer reflexões realistas de possibilidades. Avalia-se sempre a relação terapêutica e o seu fortalecimento constante através de empatia, valorização das queixas, técnicas e tarefas de casas.

Considerada uma modalidade eficaz com estrutura útil para lidar com o problema através da reestruturação de esquemas e modificação de pensamentos e comportamentos, 
antes desadaptativos e disfuncionais, frente a contingências estressoras e a prevenção de recaída, além de educar o paciente a ser mais independente para lidar de forma saudável e funcional em momentos e contextos diversos, diante de suas crises, trabalhando e reforçando a prática de respostas adaptativas.

\section{CONSIDERAÇÕES FINAIS}

Não se pretende esgotar o assunto, uma vez que diante de um tema tão complexo, ainda há poucas discussões teóricas e modos de enfrentamentos escassos. Também não se pretende estabelecer uma única abordagem teórica para discussão, tratamento e manutenção dos resultados. No presente texto foi apontado a TCC como uma abordagem teórica e prática eficaz para os pacientes portadores do TPB, entretanto, há muito ainda o que pesquisar e trazer à tona discussões para o aperfeiçoamento em prol do paciente que enfrenta os dissabores de tal psicopatologia.

Tendo isso em vista, que a presença da sintomatologia do TPB vai muito mais além do que o reducionismo da automutilação, fica claro a necessidade de aprofundamento em teorias cientificas por parte dos profissionais psicólogos, psiquiatras e pesquisadores da área sobre o citado transtorno. Diante da gravidade do que seja o transtorno de personalidade de borderline e os prejuízos reais e gradativos na vida dos portadores, percebe-se a importância do psicodiagnóstico e sua realização de modo sistemático e comprometido por parte do profissional, com o objetivo claro da redução de danos e melhoria da qualidade de vida.

O enfretamento do transtorno não é realizado de forma individual, mas em conjunto, incluindo o profissional, o paciente e familiares, de forma sistemática e seguindo um percurso estruturado que requer paciência e dedicação; levando-se em consideração que o profissional está diante, por um lado, de um paciente com personalidade limítrofe e que precisa ser acolhido empaticamente e, por outro lado, de familiares vitimizados muitas vezes pelas hostilidades e agressões de seus doentes e que já estão muitas vezes desacreditados do restabelecimento da paz em seus ambientes.

A psicoterapia na abordagem da TCC, cujo objetivo é minimizar o sofrimento e prejuízos causados aos portadores, seus familiares e seu meio social, é a forma mais humanizada de ver o paciente em sua totalidade. Sendo assim, não há minimização eficaz de sofrimento sem ajuda psicológica adequada. Portanto, é imprescindível por parte do 
profissional, uma escuta acurada para a aplicação teórica e prática adequada no momento certo, assertividade nas intervenções baseadas em evidências principalmente nos momentos de crises agudas, levando sempre em consideração as queixas iniciais, a demanda latente e os prejuízos estabelecidos e os iminentes, sempre com o intuito de redução de danos, promoção da saúde e prevenção de recaídas.

Infere-se, portanto, que diante do exposto é fundamental que o profissional tenha um conhecimento teórico-prático adequado para lidar com queixas e demandas, muitas vezes latentes a princípio, tenha um repertório adequado teórico, de empatia, de acolhimento e de escuta diferenciada para que haja progresso durante o percurso do tratamento. Se tem cura ou não o citado transtorno, ainda não se sabe efetivamente, uma vez que há discordâncias entre alguns autores, porém, no presente artigo discorre que a psicoterapia em TCC é um excelente aliado para a redução de danos, a prevenção de recaídas e, sequentemente a melhoria da qualidade de vida tanto para os pacientes em sofrimento quanto para seu entorno.

Assim, o texto em questão responde de forma adequada aos questionamentos iniciais sobre a eficácia da psicoterapia na abordagem da terapia cognitiva comportamental em suas várias nuances e desafios impostos pela própria psicopatologia que acomete os seus portadores.

\section{REFERÊNCIAS}

CARVALHO, Marcelo da Rocha. Conceitualização em Terapia Cognitiva - SlideShare/ Marcelo da Rocha Carvalho. Disponível em:

〈https://www.slideshare.net/marcelodcarvalho/ conceitualizacao-em-terapia-cognitiva $>$ Acesso em 18 de dez. 2020

DALGALARRONDO, Paulo; VILELA, Wolgrand Alves. Transtorno borderline: história e atualidade. Rev. latinoam. psicopatol. fundam. , São Paulo, v. 2, n. 2, pág. 52-7I, junho de I999. Disponível $<$ http://www.scielo.br/scielo.php?script=sci_arttext\&pid=Si4I547141999000200052\&lng=en\&nrm=iso >. Acesso em I4 de fevereiro de 2021.

DEMO, Pedro. Pesquisa e construção do conhecimento: metodologia científica no caminho de Habermas. Rio de Janeiro: Tempo Brasileiro, 2000.

FREITAS, Partinobre Brito; RECH, Terezinha. O uso da terapia cognitivocomportamental no tratamento do transtorno depressivo: uma abordagem em grupo. 
Santa Cruz do Sul: Barbaroi, n. 32, p. 98-II3, jun. 2010. Disponível em $<$ http://pepsic.bvsalud.org/scielo. php?script $=\quad$ sci_arttext\&pid=Soro4$65782010000100007 \& \operatorname{lng}=\mathrm{pt} \& \mathrm{nrm}=$ iso $>$. Acesso em I8 dez. 2020

Fronteiras da terapia cognitiva/ Paul M. Salkovskis, editor; prólogo por S. Rachman; Ana Maria Serra, organizadora da tradução brasileira. -2.ed.- São Paulo: Casa do Psicólogo, 2012.

GIL, Antônio Carlos. Como elaborar projetos de pesquisa/ Antônio Carlos Gil. - 6 ed. São Paulo: Atlas, 2017.

HAVRELHUK, Jucimar; LARANGO, Fabíola. História de Lino: a psicopatologia na perspectiva da fenomenologia e existencialismo. Rev. abordagem gestalt., Goiana,v.26,

n.I, p39-52, abr.2020. Disponível em: 〈http://pepsic.bvsalud.org/ scielo.php?script= sci_arttex\&pid=SI809-68672020000100005\&Ing=pt\&nrm=iso $>$ Acesso em 3I Jan. 202I

Instituto Beck $p$ terapia cognitiva comportamental. Bala Cynwyd, EUA, 2020. Disponível em:< https://beckinstitute.org/about-beck/history-of-cognitive-therapy/ $>$ Acesso em i2 de fev. 2021

Manual diagnóstico e estatístico de transtornos mentais [recurso eletrônico]: DSM-5 [American Psychiatric Association ; tradução: Maria Inês Corrêa Nascimento ... et al.] ; revisão técnica: Aristides Volpato Cordioli ... [et al.]. - 5. ed. - Dados eletrônicos. - Porto Alegre: Artmed, 2014. Disponível em: $<$ http://www.niip.com.br/wpcontent/uploads/2018/o6/Manual-Diagnosico-e-Estatistico-de-Transtornos-MentaisDSM-5-I-pdf.pdf> Acesso em: or de nov. 2020

MARQUES, Sofia; BARROCAS, Daniel; RIJO, Daniel. Intervenções Psicológicas na Perturbação Borderline da Personalidade: Uma revisão das Terapias de Base CognitivoComportamental. Rev. Acta Médica Portuguesa. V.30. n.4, p.309, 2017. Disponível em < http://hdl.handle.net/ io3r6/ 46676> Acesso em o7 de fev. 2021

MEDEIROS, Nat. Matias de; PEREIRA, Fernanda de Oliveira. Visão crítica da história da loucura na formação em Psicologia. PSI UNISC, [S.1.], v. 5, n. I, p. 36-50, jan. 2021.

ISSN 2527-I288. Disponível em: <https://online.unisc.br/ seer/index.php/psi/ article/view/I4536/ 9590> Acesso em: o6 fev. 2021.

MULLER, Vanessa. Transtorno da Personalidade Borderline e o Cérebro: Tratamentos Inovadores. VTM Neurodiagnóstico. Rio de Janeiro: Julho/2020. Disponível em: < https:// vtmneurodiagnost ico.com.br/2020/07/22/transtorno-da-personalidadeborderline-e-o-cerebro-tratamentos-inovadores $>$ Acesso em o7 de fev. 202I

Princípios Fundamentais. Código de Ética Profissional do Psicólogo: Brasília, ag. 2005. Disponível em: <http://site.cfp.org.br/wp-content/uploads/2012/o7/codigo-de-eticapsicologia.pdf > Acesso em: 02 de nov. 2020

Psicodiagnóstico-V [recurso eletrônico] / Jurema Alcides Cunha ... [et al.]. - 5. ed. rev. e ampl. - Dados eletrônicos. - Porto Alegre: Artmed, 2007. Disponível em: < 
http://www.brunovivas.com/

content/uploads/sites/ro/2019/o4/psicodiagnc3b3stico-v-jurema-alcides-cunha.pdf> Acesso em: 3I de out. 2020

Questões gerais sobre a avaliação psicológica: Cartilha de Avaliação Psicológica - 2013: Brasília, nov.2013. Disponível em: <https://satepsi.cfp.org.br/docs/cartilha.pdf.> Acesso em: 3I de out. 2020

SIlvA, E. C. M., GARUTTI, J. S., COSTA, R. F. Intervenção Psicológica No

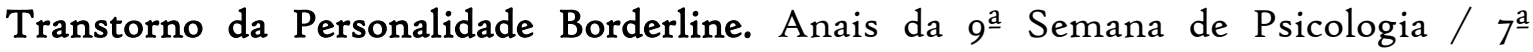
Conferência de Estudos Psicológicos / 24 a 27 de outubro de 2016, Santa Fé do Sul (SP), v.3, n.3, 2016

SILVA, N. F.; BEZERRA, E. M. Terapia cognitivo-comportamental e terapia comportamental dialética no tratamento do Transtorno da Personalidade Borderline. Hum@nae/ Questões controversas do mundo contemporâneo. São Paulo: V.15, n.I, p.3, 2021. Disponível em: $<$ file:///C:/Users/Usuarios/Downloads/776-I724-I-PB.pdf > Acesso em o6 de fev 202I

USEVICIUS, André Alves et al. Transtorno de Personalidade Borderline e a Estruturação do Self. Goiás: Repositório Institucional AEE, 2020. Disponível em: < http: //repositório.aee.edu.br/ handle/aee/9325 > Acesso em o7 de fev. 202I 